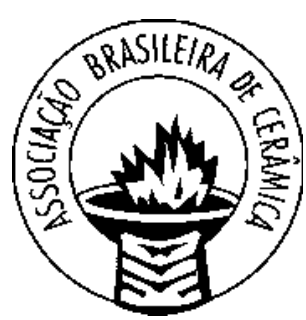

Presidente: Urames Pires dos Santos Vice-Presidente: José Carlos Bressiani

DIRETORES

Antonio Carlos de Camargo, Egon Antonio Torres Berg, José Lepri Neto, Luis Leonardo Horne Curimbaba Ferreira, Marco Antonio Pacheco Jordão, Reginaldo Muccillo, Ricardo Dutra

\section{CONSELHEIROS HONORÁRIOS}

Antonio Ermírio de Moraes, Carlos Roberto Valente da Cruz, Giulio Lattes, Nicolau de Vergueiro Forjás

\section{CONSELHO DIRETOR}

Conselheiros Eméritos:

Aguinaldo A. de Carvalho, Anselmo Ortega Boschi Alexandre R. Zandonadi, Aníbal Camillo Togni, Artur Gardelin, Celso Guimarães Pereira, Celso Monteiro de Carvalho, César da Silva Monteiro, Edgar Dutra Zanotto, Egon Antônio Torres Berg, Flávio Polo de Camargo, Gabriel W.K. Paiva Cortes, Geraldo Agosti, Gladstone Motta Bustamante, Heber Carlos Ferreira, Helena de Souza Santos, Jamil Duailibi Filho, Jorge José Nunes, José Arana Varela, José Carlos Bressiani, Luciano Barzaghi, Marcelo Ruy V. de Azevedo, Marco Antonio Pacheco Jordão, Nilziette de Mello Rodrigues, Otair Becker, Paschoal Giardullo, Pérsio de Souza Santos, Shirley Cosin, Tarcísio Sérgio Andrade, Tercílio Pozzani, Teresa Cristina Togni, Waldomiro Lunardi Pires Corrêa

\section{Conselheiros}

eleitos pelos sócios patrocinadores:

Antonio Álvaro Guedes, Gian Antonio Comazzi, José Carlos Germine, Júlio César Carvalho, Ricardo Ibanhez, Ruben Salvador Sinato, Valmy Artus G. Oliveira Jr.,

Conselheiros eleitos pelos sócios coletivos individuais:

Adalberto Benevides Magalhães Filho, Ana Helena A. Bressiani, Ana Paula M. Menegazzo, André Geraldo Rodrigues, Antonietta Tordino, Antonio Carlos da Cruz, Antônio Carlos de Camargo, Dulce Maria de Araújo Melo, Evaristo Pereira Goulart, Gelmires de Araújo Neves, Gerson Ferreira, Guilherme P. Côrtes, Ilson Piovesan, João Celso Romachelli, José Cláudio Marcon, José Octávio Armani Paschoal, Omar D.C. Afrange, Reginaldo Muccillo, Samuel M. Toffoli 2006 ,Urames Pires dos Santos, Uriel Vargas, Victor Carlos Pandolfelli, Waldir de Sousa Resende, Walter Stern, Willes Banks,

\section{CONSELHO FISCAL: SUPLENTES:}

\section{Jamil Duailibi Filho Luiz Carlos Tanno} Geraldo Agosti Roberto Tomasi

Shirley Cosin Waldir de Sousa Resende

Regional de Minas Gerais:

Wander Luiz Vasconcelos, Paulo Roberto G. Brandão.

Regional do Rio de Janeiro:

Jamil Duailibi Filho, Maria Cecília Nóbrega

Regional de Santa Catarina:

Arlindo R. Voltolini

Antônio Carlos Cardoso

Regional de São Carlos:

Dulcina Maria Pinatti F. de Souza, José de Anchieta Rodrigues

Regional da Paraíba:

Héber Carlos Ferreira, Hélio de Lucena Lira

\section{Editorial}

A cada exemplar da revista Cerâmica que é editado, maior é o número de artigos publicados. É cada vez maior o número de trabalhos submetidos para publicação. Somente durante o último Congresso Brasileiro de Cerâmica mais de quarenta trabalhos foram submetidos. Solicitamos paciência aos autores colaboradores, pois o trabalho de processamento dos trabalhos catalogação, preparação de cartas de recebimento, escolha de relatores, envio a relatores para análise e parecer, recebimento das análises dos relatores, encaminhamento aos autores, etc. - demandam um tempo enorme dada a estrutura não profissional da editoria científica. Não há secretárias, officeboys, telefonista, encarregados de receber/emitir e-mails, etc. Tudo é feito pelo Editor com a colaboração espontânea de dois pesquisadores de seu grupo de pesquisa nos laboratórios do IPEN, que se encarregam dos trabalhos exaustivos de revisão técnica e gráfica cada vez que um novo número é editado. Contamos também, é claro, com a colaboração de alguns pesquisadores de várias partes do Brasil, que dedicam parte de seu tempo na leitura e emissão de pareceres.

$\mathrm{O}$ crescimento do número de trabalhos submetidos reflete o esperado aumento da produtividade científica da área de materiais cerâmicos no Brasil, com melhoria não só na quantidade mas também na qualidade dos trabalhos, decorrente em parte de maiores financiamentos, a níveis estadual e federal, para trabalhos de pesquisa em Universidades e Institutos. Além disso, a escolha dos autores mostra que a revista Cerâmica tem desempenhado um papel de veículo para disseminação de conhecimento na área, preenchendo portanto a sua principal finalidade.

\section{O Editor}

PS: A revista Cerâmica (assim como a Cerâmica Industrial) pode ser acessada pela base de dados QUALIS - Qualificação de Periódicos, Anais, Jornais e Revistas, preparada pela CAPES - Coordenação de Aperfeiçoamento de Pessoal de Nivel Superior do Ministério da Educação. O banco de dados QUALIS é o resultado do processo de classificação dos veículos utilizados pelos programas de pós-graduação para a divulgação da produção intelectual de seus docentes e alunos. Para localizar a revista Cerâmica, uma das formas pode ser na página do Portal (www.periodicos.capes.gov.br), no box "Pesquisa por palavra do título" digitar Cerâmica, clicar em busca. A seguir, basta dar um clique no nome da revista.
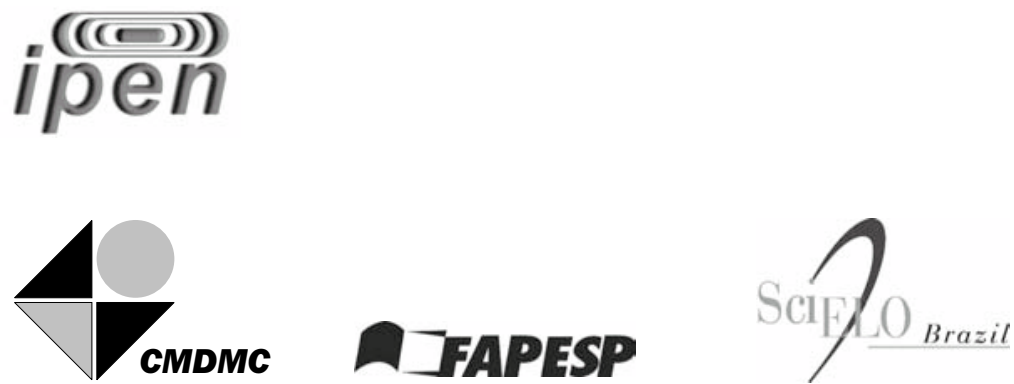